\title{
Support vector machine classifier for prediction of the metastasis of colorectal cancer
}

\author{
JIAJUN ZHI, JIWEI SUN, ZHONGCHUAN WANG and WENJUN DING \\ Department of Colorectal Surgery, Xinhua Hospital Affiliated to Shanghai Jiao Tong University School of Medicine, \\ Shanghai 200092, P.R. China
}

Received December 31, 2016; Accepted December 13, 2017

DOI: $10.3892 /$ ijmm.2018.3359

\begin{abstract}
Colorectal cancer (CRC) is one of the most common cancers and a major cause of mortality. The present study aimed to identify potential biomarkers for CRC metastasis and uncover the mechanisms underlying the etiology of the disease. The five datasets GSE68468, GSE62321, GSE22834, GSE14297 and GSE6988 were utilized in the study, all of which contained metastatic and non-metastatic CRC samples. Among them, three datasets were integrated via meta-analysis to identify the differentially expressed genes (DEGs) between the two types of samples. A protein-protein interaction (PPI) network was constructed for these DEGs. Candidate genes were then selected by the support vector machine (SVM) classifier based on the betweenness centrality (BC) algorithm. A CRC dataset from The Cancer Genome Atlas database was used to evaluate the accuracy of the SVM classifier. Pathway enrichment analysis was carried out for the SVM-classified gene signatures. In total, 358 DEGs were identified by meta-analysis. The top ten nodes in the PPI network with the highest $\mathrm{BC}$ values were selected, including cAMP responsive element binding protein 1 (CREB1), cullin 7 (CUL7) and signal sequence receptor 3 (SSR3). The optimal SVM classification model was established, which was able to precisely distinguish between the metastatic and non-metastatic samples. Based on this SVM classifier, 40 signature genes were identified, which were mainly enriched in protein processing in endoplasmic reticulum (e.g., SSR3), AMPK signaling pathway (e.g., CREB1) and ubiquitin mediated proteolysis (e.g., FBXO2, CUL7 and $U B E 2 D 3$ ) pathways. In conclusion, the SVM-classified genes, including CREB1, CUL7 and SSR3, precisely distinguished the metastatic CRC samples from the non-metastatic ones. These
\end{abstract}

Correspondence to: Dr Wenjun Ding, Department of Colorectal Surgery, Xinhua Hospital Affiliated to Shanghai Jiao Tong University School of Medicine, 1665 Kongjiang Road, Shanghai 200092, P.R. China

E-mail: wow_dingwenjun@163.com

Key words: colorectal cancer, metastasis, microarray, meta-analysis, support vector machine, classification genes have the potential to be used as biomarkers for the prognosis of metastatic CRC.

\section{Introduction}

Colorectal cancer (CRC) is one of the most lethal diseases worldwide, and it is estimated to account for $>9 \%$ of all cases of cancer. The incidence of CRC varies according to geographical location, and the majority of cases occur in developed countries (1). Based on epidemiological data, it was estimated that 136,830 individuals would be diagnosed with CRC and 50,310 would succumb to the disease in the USA in 2014 (2).

Genetic factors have been established as major regulators that affect CRC pathogenesis. Germline mutations of susceptibility genes, such as adenomatous polyposis coli, MutL homolog 1, MutL homolog 2 and the three loci recently identified near to the genes paired like homeodomain 1, cyclin D2 and hydroxyacid oxidase 1, are considered to be tightly associated with CRC risk (3). SRY-box containing gene 17 is a transcription factor (TF) that functions as an inhibitor in the Wnt pathway, and its abnormal expression caused by promoter hypermethylation may influence CRC development (4). The activation of nuclear factor- $\kappa \mathrm{B}$ signaling and its regulated genes also serve important roles in the promotion of CRC progression (5). Metastasis is the most common cause of cancer-associated mortality, and accounts for $\sim 90 \%$ of all cancer deaths (6). Patients with metastatic CRC have a poor 5-year survival rate of $<10 \%$ (7). A number of studies have investigated the molecular mechanisms of metastatic CRC. For instance, the overexpression of AKT serine/threonine kinase 2 has been indicated to be a causative factor for CRC metastasis (8). Another study identified several metastasis-associated genes in CRC, which mainly participate in extracellular matrix interactions and cell signaling functions, and include integrin subunit $\beta 1$, integrin subunit $\beta 5$, collagen type V $\alpha 1$ and secreted phosphoprotein 1 (9). A further study indicated that metastatic gene signatures, such as chemokine (C-X-C Motif) receptor 7, adenylate kinase 1 and early growth response 1 are able to predict the risk of recurrence and mortality in patients with CRC (10). Despite these profound findings, the etiology of CRC metastasis remains obscure.

The support vector machine (SVM) classifier is a kernel algorithm that bases its analysis on data obtained only through dot-products. The SVM classifier is widely applied in 
bioinformatics due to its high accuracy, and has the ability to identify the multivariate statistical properties of data that distinguish between two different groups $(11,12)$. Henneges et al $(13)$ demonstrated that the SVM classifier, in combination with liquid chromatography ion trap mass spectrometry, is a promising tool for crucial gene predictions in non-invasive breast cancer. In addition, another study using SVM established a model that was able to discriminate normal samples from those of CRC patients; via this classification method, several biomarkers were predicted, including cadherin 3 , claudin 1 and interleukin-8 (14). However, to the best of our knowledge, there have been no previous reports regarding the application of the SVM classifier to CRC metastasis.

Therefore, the present study was performed using the SVM method to classify metastatic and non-metastatic CRC samples. Three datasets were integrated using meta-analysis and an additional dataset from The Cancer Genome Atlas (TCGA) database was utilized to validate the precision of the SVM classifier. Several bioinformatic methods were then carried out to reveal function and pathway information of the identified SVM-classified signature genes, on the basis of which a comprehensive evaluation of the metastatic mechanisms in $\mathrm{CRC}$ was conducted and novel biomarkers identified.

\section{Materials and methods}

Data resources and pretreatment. The Gene Expression Omnibus (GEO; http://www.ncbi.nlm.nih.gov/geo) database was searched for all eligible open public datasets with the key search terms of 'colon cancer' and 'homo sapiens'. Datasets that satisfied the following criteria were included in the study: i) The data comprised gene expression profiles; ii) the data were associated with CRC and metastasis; iii) information on samples from patients with CRC and controls was elaborated. Based on these selection criteria, five microarray datasets, GSE68468 (15), GSE62321 (16), GSE22834 (17), GSE14297 (18) and GSE6988 (19) were included in the present study.

Among these datasets, GSE68468 and GSE62321 were from the same platform, Affymetrix HG-U133 arrays (Thermo Fisher Scientific, Inc., Waltham, MA, USA). GSE68468 consisted of 240 CRC samples, of which 47 were metastatic and 193 were non-metastatic. GSE62321 comprised a total of 39 CRC samples, including 19 metastatic and 20 non-metastatic samples. For these two datasets, raw data in the CEL format was downloaded from the GEO database, followed by background correction and normalization using the Microarray Suite and quantiles, respectively $(20,21)$. The median method was used for the supplementation of missing values. These pretreatments were performed using the Affy package in $\mathrm{R}$ version 1.42 .3 (http://www.bioconductor.org/ packages/release/bioc/html/affy.html).

Regarding the remaining three datasets, GSE22834 was obtained from the Stanford Microarray Database print platform (Stanford University, Stanford, CA, USA), and consisted of 63 CRC samples (32 metastatic and 31 non-metastatic); GSE14297 was derived from the Illumina human-6 v2.0 expression beadchip (extended) (Illumina, Inc., San Diego, CA, USA), and included 36 CRC samples (18 metastatic and 18 non-metastatic); and GSE6988 was from the Human 17K
cDNA-GeneTrack platform (Genomic Tree, Inc., Daegeon, Korea), and comprised 53 CRC samples (33 metastatic and 20 non-metastatic). For these three datasets, raw data in the txt format was downloaded in the respective platform. In each annotation platform, the probe identification number was transformed into gene expression symbols. Probes that had a vacancy were deleted, and multiple probes that corresponded to a single gene were averaged to obtain the gene expression value. The Linear Models for Microarray Analysis (limma; http://www.bioconductor.org/packages/release/bioc/html/limma.html) package version 3.22.1 was then used to normalize the data (22).

Selection of differentially expressed genes (DEGs) using meta-analysis. To eliminate the bias from different platforms, the MetaQC package version 0.1.13 was utilized to perform quality control of the different datasets, in combination with principal component analysis and standardized mean rank (23). The standards in MetaQC included: i) Internal quality control, which was used to determine the structural homogeneity of gene expression values among different datasets; ii) external quality control, which was used for the consistency testing of gene expression in a pathway database; iii) accuracy quality control, which was used to determine the accuracy of a differentially DEG or recognition of a pathway; iv) consistency quality control of a DEG and pathway.

Following quality control, MetaDE.ES in the MetaDE package [(23) https://cran.r-project.org/web/packages/ MetaQC/index.html] was utilized to identify DEGs in the integrated dataset. First, the heterogeneity of the expression of each gene in different platforms was detected based on parameters including $\tau^{2}$, the $Q$ value and Qpval $\left(\tau^{2}=0\right.$ indicates homogeneity and a lack of bias; a Q statistic obeying the $\chi^{2}$ test with a freedom of K-1 and Qpval >0.05 indicate homogeneity and a lack of bias). DEGs between the different groups in this integrated dataset were then selected, and the P-value and false discovery rate (FDR) were obtained. FDR $<0.05$ indicated a significant difference. Thresholds for DEGs among different groups in the present study were $\tau^{2}=0$, Qpval $>0.05$ and FDR $<0.05$. Thereafter, these DEGs were subjected to bi-directional hierarchical clustering analysis using the pheatmap R package version 1.0.2 (http://cran.r-project.org/ web/packages/pheatmap/index.html).

Construction of a protein-protein interaction (PPI) network and its topological properties. Information in the human protein reference database (HPRD; http://www.hprd.org/) was integrated with that in the Biological General Repository for Interaction Datasets (BioGRID; http://www.thebiogrid. org) $(24,25)$ to construct a PPI network for the identified DEGs. The network was visualized using Cytoscape software version 3.6.0 (http://cytoscape.org/).

The betweenness centrality $\left(\mathrm{BC}\right.$ or $\mathrm{C}_{\mathrm{B}}$ ) algorithm was used to reflect the topological property of each gene in this network and to optimize candidate genes (26). The BC value of each DEG was calculated based on the following formula:

$$
C_{B}(v)=\sum_{t \neq v \neq u \in V} \frac{\sigma_{s t}(v)}{\sigma_{s t}}
$$

In the formula, $\mathrm{v}, \mathrm{s}$ and $\mathrm{t}$ denote three nodes (protein production of DEGs) in the PPI network, $\sigma_{\mathrm{st}}$ is the number of shortest paths 
from 's' to 't', and $\sigma_{\mathrm{st}}(\mathrm{v})$ reflects the number of $\sigma_{\mathrm{st}}$ that pass the node ' $v$ '. The $\mathrm{BC}$ value varies from $0-1$, and the greatest value indicates the highest centrality of a node in the PPI network.

Training of the optimal SVM classification model and performance evaluation. The DEGs were sorted in descending order based on their $\mathrm{BC}$ values, and for those ranked at 10 to 100 , the dataset that conformed to the quality control and had the largest sample number was set as the training dataset to perform training of the optimal SVM classification model, until it could absolutely distinguish one sample from another (27). DEGs obtained by this SVM classifier were then further investigated using bi-directional hierarchical clustering analysis, with visualization using the aforementioned heatmap software. Afterwards, the remaining datasets were taken as the validation datasets to evaluate the accuracy of the optimal SVM classifier.

Validation via an individual dataset. A CRC dataset that was downloaded from the TCGA database (https:// cancergenome.nih.gov/), with the accession number TCGA COAD_G4502A_07_3-2015-02-24 (level 3), was used for the validation. The dataset included a total of 193 specimens, and 90 of them had available clinical information, including 14 and 76 cases with and without the appearance of additional tumors, respectively.

Enrichment analysis. Kyoto Encyclopedia of Genes and Genomes (KEGG; http://www.genome.jp/kegg/pathway.html) pathway enrichment analysis was carried out for these DEGs to identify their potential pathways, using Fisher's exact test based on the following formula:

$$
p=1-\sum_{i=0}^{x-1} \frac{\left(\begin{array}{c}
M \\
i
\end{array}\right)\left(\begin{array}{c}
N-M \\
K-i
\end{array}\right)}{\left(\begin{array}{l}
N \\
K
\end{array}\right)}
$$

In the formula, $\mathrm{N}$ represents total gene counts in the whole genome, $\mathrm{M}$ indicates gene counts in the pathways, $\mathrm{K}$ denotes DGE counts, and $p$ represents the probability of $\geq x$ of the $\mathrm{K}$ DEGs being enriched in the pathway.

\section{Results}

DEGs detected by meta-analysis. Quality control conducted using MetaQC indicated that the GSE22834 and GSE6988 datasets had relatively low quality, compared with the others (Table I). In addition, GSE22834 markedly deviated from the other four datasets, and GSE6988 also exhibited evident bias (Fig. 1). For these reasons, these two datasets were excluded. The remaining three datasets were selected for data integration via meta-analysis. The parameters pval, FDR, $\tau^{2}$, Qpval and Qval were calculated using MetaDE. Based on the aforementioned selection criteria, a total of 358 DEGs were identified by integrating the three datasets, and the top 10 DEGs are listed in Table II. A heat map of the gene expression of the 358 genes is presented in Fig. 2.

PPI network of the DEGs. By integrating protein information in the HPRD database with that in BioGRID, interactions among
Table I. Quality control results of the five datasets.

\begin{tabular}{llllllllr}
\hline Dataset & IQC & EQC & CQCg & CQCp & AQCg & AQCp & SMR \\
\hline GSE68468 & 5.19 & 3.28 & 69.15 & 103.59 & 27.46 & 56.31 & 2.13 \\
GSE62321 & 3.76 & 3.15 & 56.7 & 148.66 & 33.78 & 47.61 & 3.59 \\
GSE22834 & 0.21 & 0.67 & 0.01 & 0.27 & 0.83 & 1.98 & 13.87 \\
GSE14297 & 7.65 & 4.32 & 1.92 & 59.62 & 21.19 & 2.39 & 6.02 \\
GSE6988 & 0.03 & 1.19 & 0.86 & 0.53 & 1.73 & 1.96 & 8.62
\end{tabular}

IQC, internal quality control; EQC, external quality control; CQCg, consistency quality control of gene; CQCp, consistency quality control of pathway; $\mathrm{AQCg}$, accuracy quality control of gene; $\mathrm{AQCp}$, accuracy quality control of pathway; SMR, standardized mean rank.

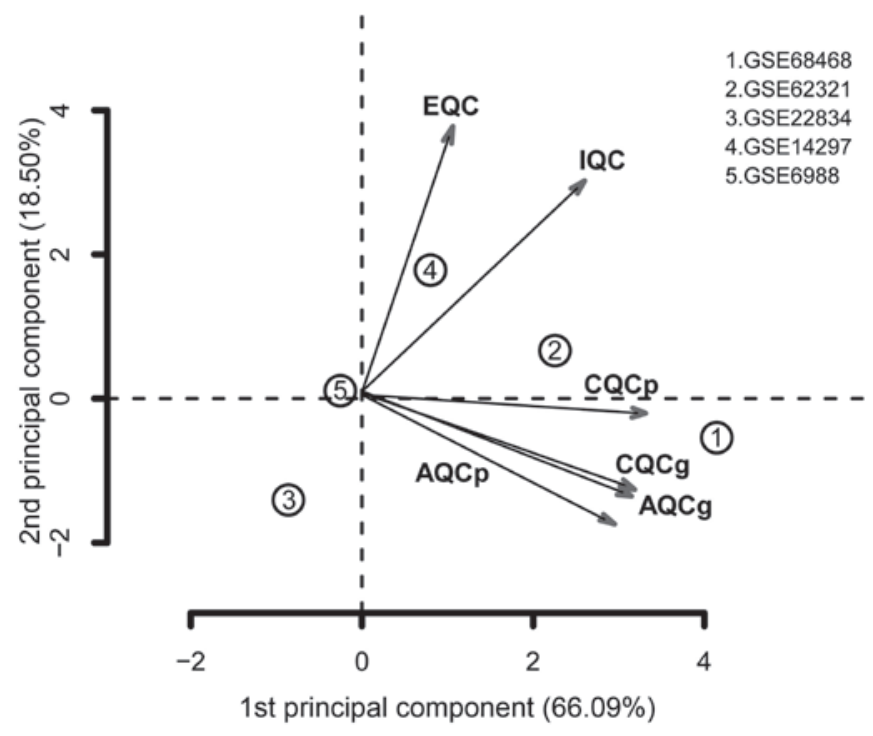

Figure 1. Quality control of the five datasets via MetaQC. Numbers 1-5 denote the five datasets. IQC, internal quality control; EQC, external quality control; $\mathrm{CQCg}$, consistency quality control of gene; CQCp, consistency quality control of pathway; AQCg, accuracy quality control of gene; AQCp, accuracy quality control of pathway.

the 358 DEGs were extracted to form a PPI network, which contained 162 nodes and 193 interactions (Fig. 3).

DEGs optimized by BC of the network. Based on the BC algorithm, the $\mathrm{BC}$ value of each node was obtained, and the top 10 nodes were: BCL6 corepressor; coatomer protein complex subunit $\beta 2$; cAMP responsive element binding protein 1 (CREB1); myosin heavy chain 11; family with sequence similarity 3 member C; InaD-like (also known as PATJ, crumbs cell polarity complex component); RAB32, member RAS oncogene family; translocation of outer mitochondrial membrane 22; cullin 7 (CUL7); and signal sequence receptor 3 (SSR3). Detailed information is listed in Table III.

Optimal SVM classification model and performance evaluation results. GSE68468, which includes 47 metastatic and 193 non-metastatic CRC samples, was used as the training 

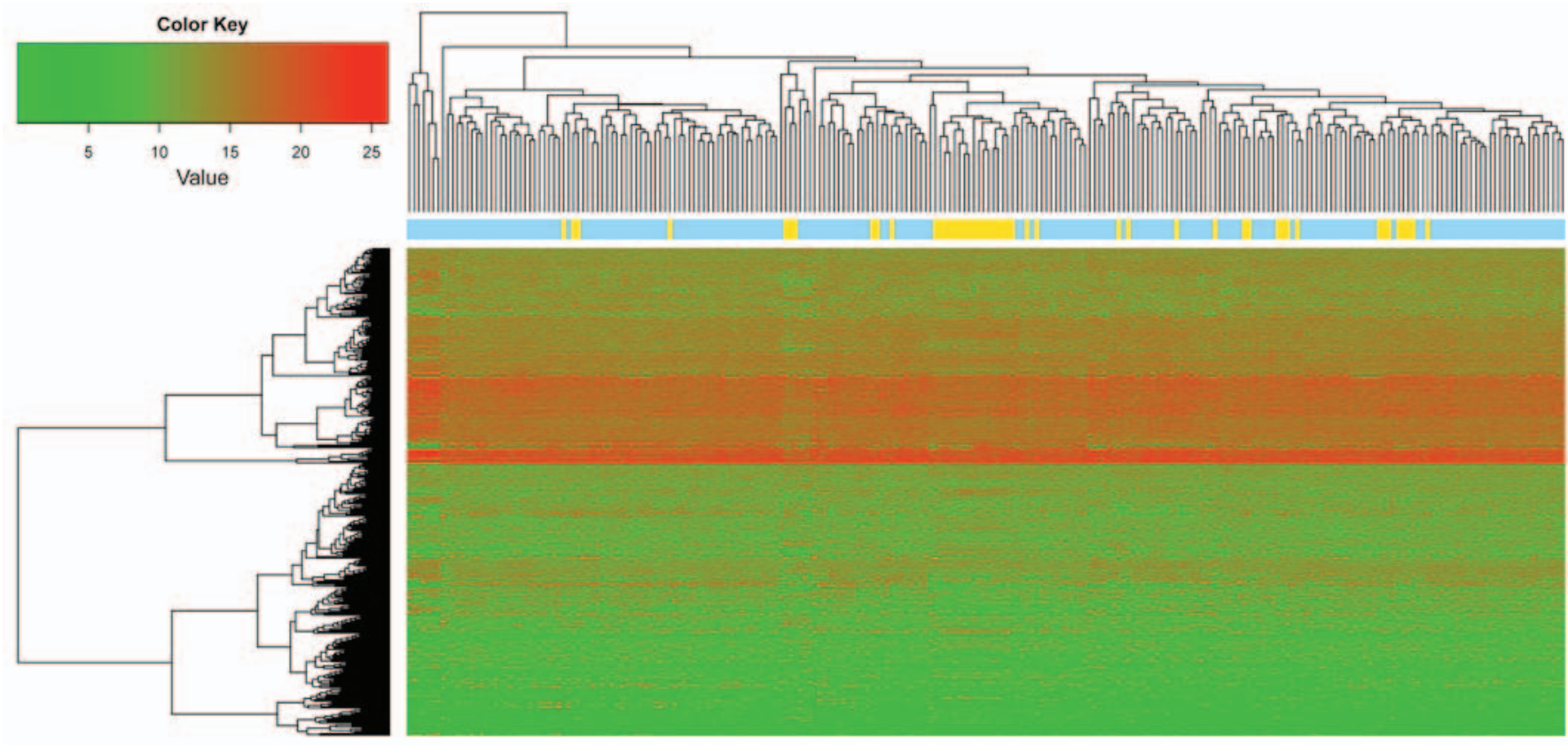

Figure 2. Heat map of the gene expression of the 358 differentially expressed genes in metastatic and non-metastatic colon cancer samples. Red indicates high expression and green indicates low expression, yellow represents metastatic samples and blue represents non-metastatic samples.

Table II. Top 10 differentially expressed genes identified via meta-analysis of the three integrated datasets.

\begin{tabular}{llllll}
\hline Gene & P-value & FDR & $\mathrm{Q}$ & $\mathrm{Qp}$ & $\tau^{2}$ \\
\hline MCF2L & $1.00 \times 10^{-20}$ & $3.45 \times 10^{-18}$ & 1.7104 & 0.4252 & 0 \\
TCF21 & $1.00 \times 10^{-20}$ & $3.45 \times 10^{-18}$ & 0.9410 & 0.6247 & 0 \\
FGD6 & $1.00 \times 10^{-20}$ & $3.45 \times 10^{-18}$ & 0.9375 & 0.6258 & 0 \\
MED28 & $1.00 \times 10^{-20}$ & $3.45 \times 10^{-18}$ & 0.7498 & 0.6874 & 0 \\
PRDM1 & $1.00 \times 10^{-20}$ & $3.45 \times 10^{-18}$ & 0.7372 & 0.6917 & 0 \\
TMED10 & $1.00 \times 10^{-20}$ & $3.45 \times 10^{-18}$ & 0.6972 & 0.7057 & 0 \\
F5 & $1.00 \times 10^{-20}$ & $3.45 \times 10^{-18}$ & 0.4327 & 0.8054 & 0 \\
NUMA1 & $1.00 \times 10^{-20}$ & $3.45 \times 10^{-18}$ & 0.2751 & 0.8715 & 0 \\
ELOVL6 & $3.62 \times 10^{-6}$ & $7.69 \times 10^{-4}$ & 1.9948 & 0.3688 & 0 \\
DLD & $3.62 \times 10^{-6}$ & $7.69 \times 10^{-4}$ & 1.8035 & 0.4059 & 0 \\
\hline
\end{tabular}

FDR, false discovery rate; MCF2L, MCF.2 cell line derived transforming sequence like; TCF21, transcription factor 21; FGD6, FYVE RhoGEF and PH domain containing 6; MED28, mediator complex subunit 28; PRDM1, PR/SET domain 1; TMED10, transmembrane p24 trafficking protein 10; F5, coagulation factor 5; NUMA1, nuclear mitotic apparatus protein 1; ELOVL6, ELOVL fatty acid elongase 6 ; DLD, dihydrolipoamide dehydrogenase.

dataset to perform SVM classification training, until the SVM classification was able to completely distinguish the two types of sample. In the training process, as the number of DEGs was increased from the top 10 to the top 100, the precision of the SVM classification increased from 98 to $100 \%$. Notably, the precision remained at $100 \%$ as the number of DEGs increased from the top 40 to the top 100. Therefore, the DEGs whose $\mathrm{BC}$ value ranked within the top 40 were selected to build the
SVM classification model with a strong ability to distinguish metastatic samples from non-metastatic ones. The scattergram is shown in Fig. 4.

To determine whether the SVM classification constructed using the top 40 genes was repeatable, two other datasets, GSE62321 and GSE14297 were used to test the precision of the classification. As shown in Fig. 5, this SVM classification was clearly able to distinguish between metastatic and non-metastatic samples in these two datasets.

Validation results. The CRC dataset downloaded from TCGA was set as the individual validation dataset, which was used to testify the performance evaluation of the SVM classification. As a result, 13 metastatic and 76 non-metastatic samples were correctly identified. Only 1 sample was wrongly classified and the area under the curve was 0.997 (Fig. 6).

Pathways of the 40 DEGs. On the basis of Fisher's exact test, five pathways were identified for these 40 DEGs (Table IV), namely protein processing in endoplasmic reticulum (ER) [e.g., F-box protein 2 (FBXO2), DnaJ heat shock protein family (Hsp40) member C10 (DNAJC10) and SSR3], AMP-activated protein kinase (AMPK) signaling pathway [e.g., protein kinase AMP-activated non-catalytic subunit $\beta 2$ (PRKAB2), phosphofructokinase, platelet $(P F K P)$ and $C R E B 1]$, dorso-ventral axis formation [e.g., mitogen-activated protein kinase 1 (MAPK1) and notch 1 (NOTCH1)], ubiquitin mediated proteolysis [e.g., $F B X O 2, C U L 7$ and ubiquitin conjugating enzyme E2 D3 (UBE2D3)] and prion diseases (e.g., MAPK1 and NOTCH1).

\section{Discussion}

The present study identified 40 SVM-classified signature genes in metastatic CRC, including CREB1, CUL7 and SSR3, 
Table III. Top 10 differentially expressed genes ranked by their betweenness centrality value.

\begin{tabular}{|c|c|c|c|c|c|c|c|c|}
\hline Gene & $\mathrm{BC}$ & Exp & Degree & P-value & FDR & Q & Qp & $\tau^{2}$ \\
\hline$B C O R$ & 1 & 1 & 2 & $1.41 \times 10^{-2}$ & 0.1337 & 0.1198 & 0.9418 & 0 \\
\hline СОРВ2 & 1 & 0 & 2 & $6.28 \times 10^{-3}$ & 0.0845 & 0.8227 & 0.6627 & 0 \\
\hline CREB 1 & 1 & 0 & 4 & $2.44 \times 10^{-2}$ & 0.1812 & 0.6522 & 0.7217 & 0 \\
\hline МYHII & 1 & 0 & 2 & $7.17 \times 10^{-4}$ & 0.0236 & 0.8618 & 0.6499 & 0 \\
\hline$F A M 3 C$ & 0.7 & 0 & 3 & $3.82 \times 10^{-2}$ & 0.2279 & 0.0720 & 0.9646 & 0 \\
\hline$I N A D L$ & 0.6667 & 1 & 2 & $3.26 \times 10^{-5}$ & 0.0030 & 1.6994 & 0.4275 & 0 \\
\hline$R A B 32$ & 0.6667 & 0 & 3 & $3.02 \times 10^{-2}$ & 0.1990 & 0.4374 & 0.8036 & 0 \\
\hline TOMM22 & 0.6 & 0 & 2 & $2.54 \times 10^{-5}$ & 0.0028 & 1.6978 & 0.4279 & 0 \\
\hline CUL7 & 0.4595 & 1 & 16 & $6.92 \times 10^{-4}$ & 0.0234 & 1.0330 & 0.5966 & 0 \\
\hline SSR3 & 0.4 & 0 & 2 & $1.04 \times 10^{-3}$ & 0.0291 & 1.5003 & 0.4723 & 0 \\
\hline
\end{tabular}

BC, betweenness centrality; FDR, false discovery rate; BCOR, BCL6 corepressor; COPB2, coatomer protein complex subunit $\beta$ 2; CREB1, cAMP responsive element binding protein 1; MYH11, myosin heavy chain 11; FAM3C, family with sequence similarity 3 member C; INADL, InaD-like; RAB32, RAB32, member RAS oncogene family; TOMM22, translocation of outer mitochondrial membrane 22; CUL7, culin 7; SSR3, signal sequence receptor 3 .

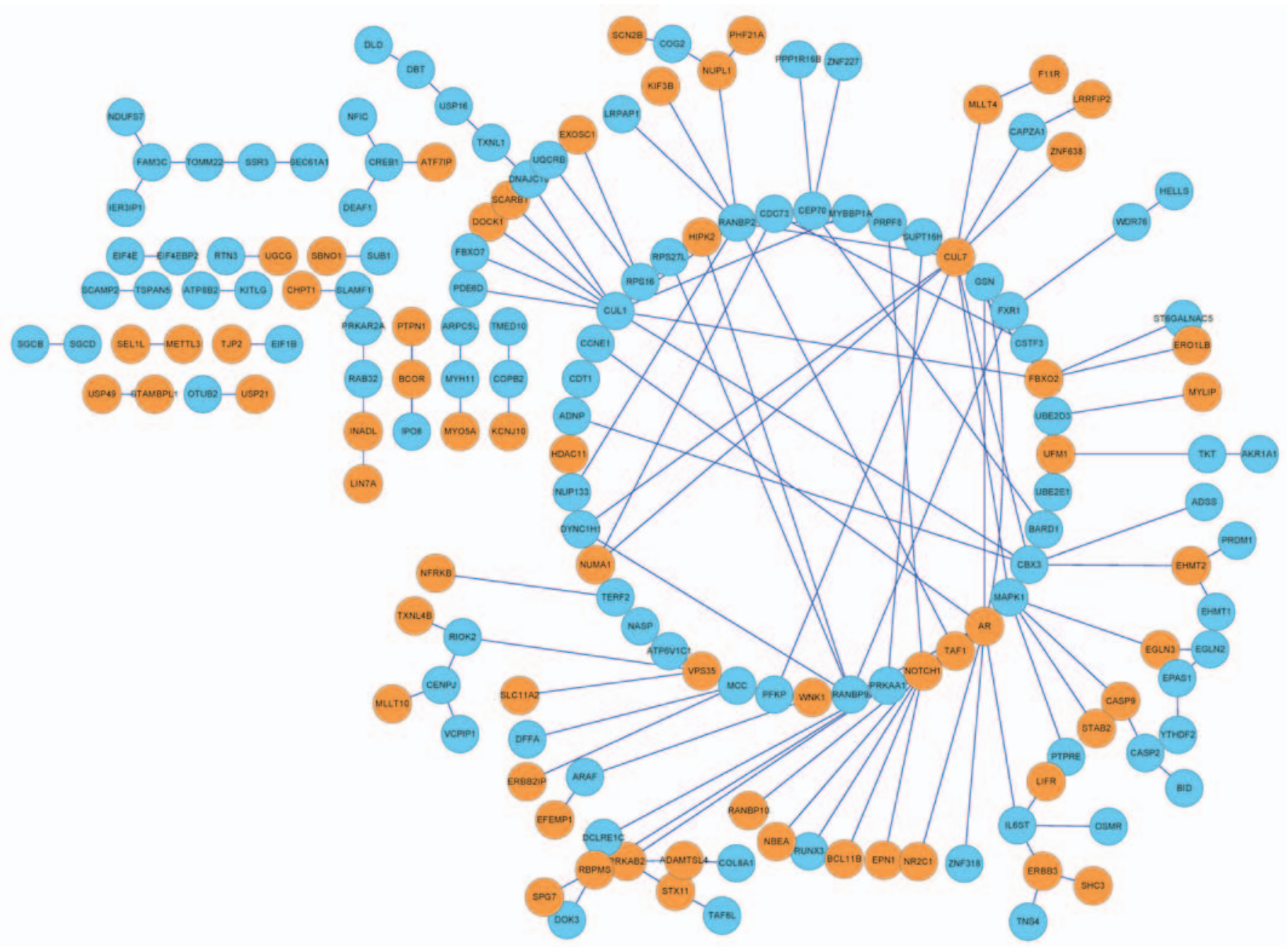

Figure 3. Protein-protein interaction network of the differentially expressed genes. Orange indicates upregulated genes and blue represents downregulated genes in metastatic compared with non-metastatic colon cancer samples. Lines between two nodes represent interactions between them.

which were significantly enriched in protein processing in ER, AMPK signaling pathway and ubiquitin mediated proteolysis functions. The precision of the SVM-classified 40 gene signatures was as high as $100 \%$, and the validation using a dataset 

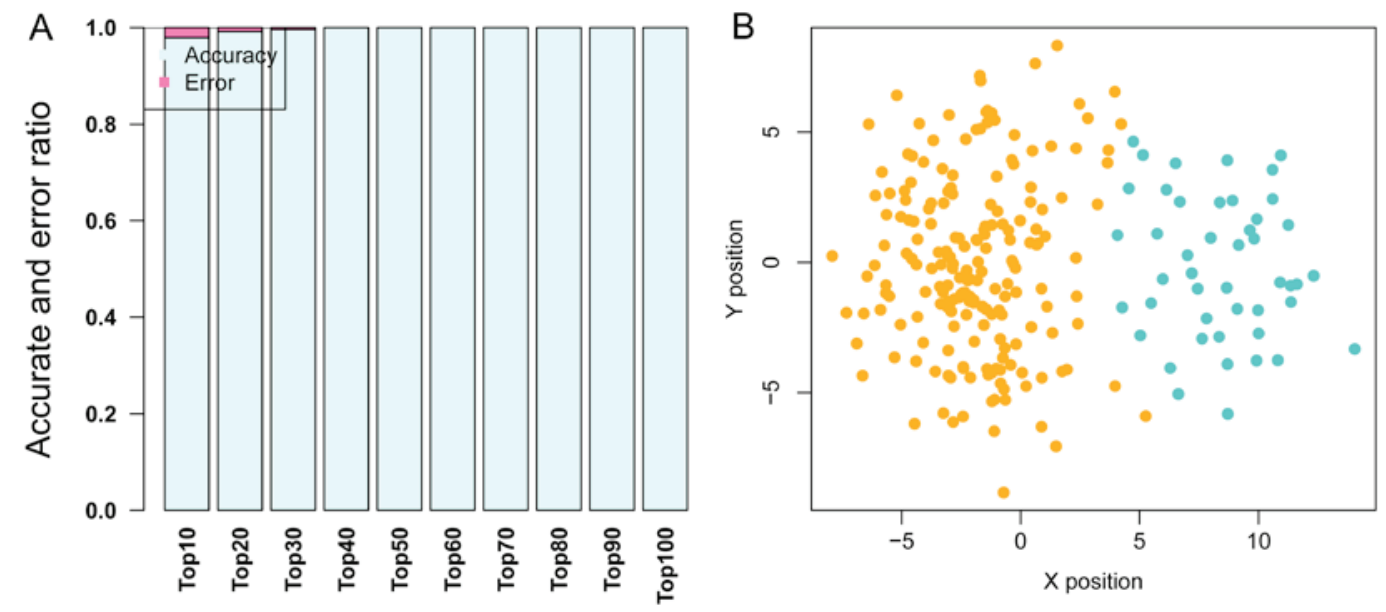

Figure 4. SVM classification and the performance evaluation result. (A) Accurate and error ratios of different training SVM classifications based on different signature genes. Red denotes error ratio and blue represents accurate ratio. (B) Scattergram based on SVM classification on different kinds of samples. Orange represents non-metastatic samples and blue represents metastatic samples. SVM, support vector machine.
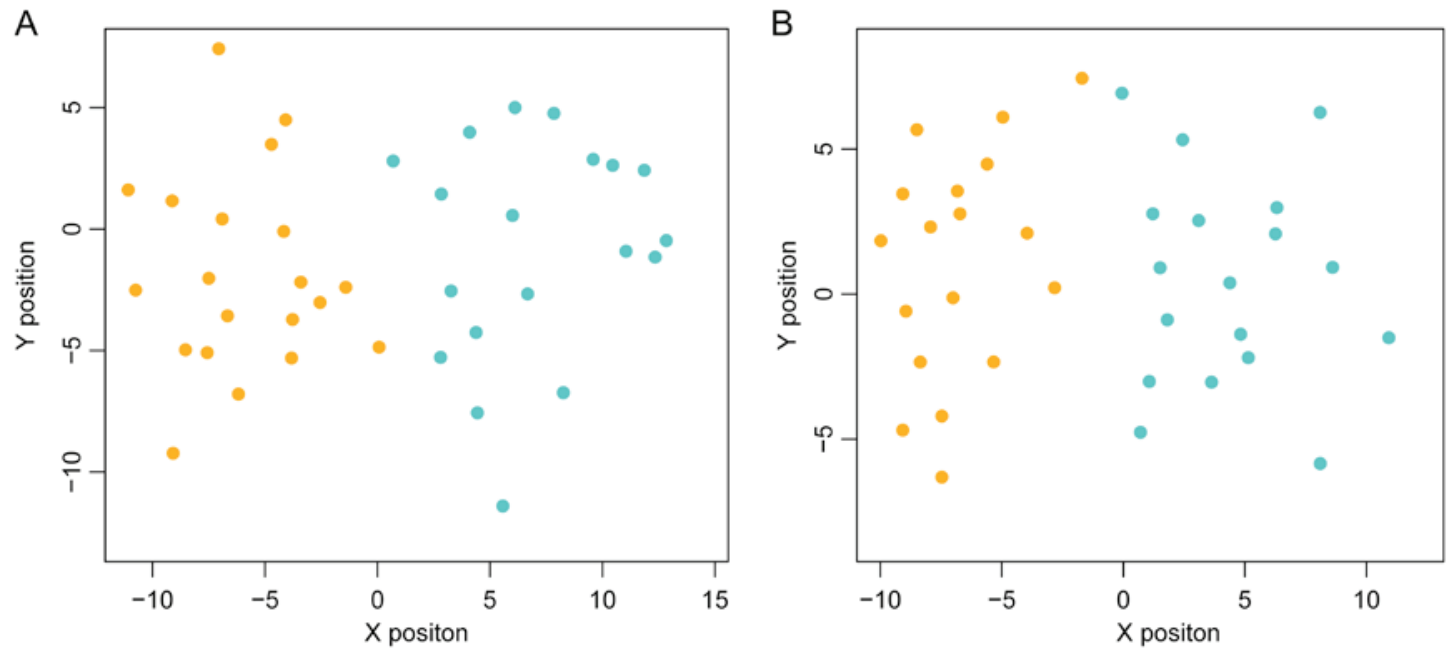

Figure 5. Scattergram based on support vector machine classification of different samples in two datasets. (A) GSE62321 and (B) GSE14297 datasets. Orange represents non-metastatic samples and blue represents metastatic samples.

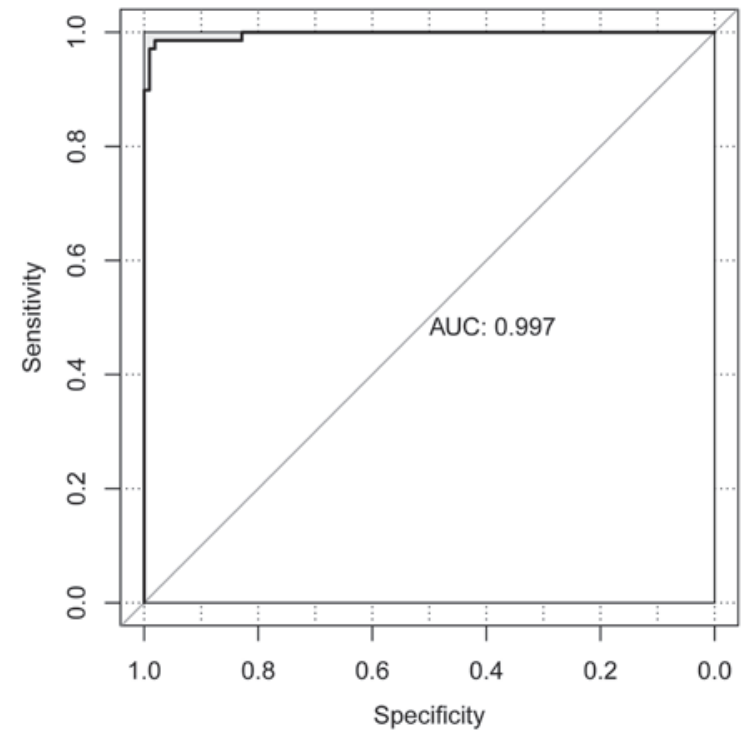

Figure 6. Receiver operating characteristic curve of support vector machine classification on individual validation datasets. AUC, area under the curve. from TCGA indicated that the majority of the metastatic and non-metastatic samples could be clearly distinguished from each other using these 40 genes.

CREB1 is a TF that belongs to the leucine zipper family. The CREBI gene is reported to increase the proliferation of CRC cells, while the knockdown of CREBI inhibits this process (28). In addition, multiple microRNAs (miRs) function as tumor suppressors in CRC development through targeting this gene, including miR-9, miR-34b and miR-200b (29). A soluble resistance-related calcium binding protein, sorcin, has been demonstrated to increase the metastasis of CRC (30). Notably, the overexpression of sorcin activates the CREB pathway by increasing the phosphorylation of CREB1 (30), which implicates the expression of CREBI in CRC metastasis, as predicted in the present study. AMPK is a heterotrimeric protein kinase that serves as a metabolic master switch. AMPK induces apoptosis in the development of CRC, and resveratrol is reported to exert therapeutic effects via inhibition of the AMPK signaling pathway (31). In the present study, CREBI was significantly enriched in the AMPK signaling pathway, 
Table IV. Pathway enrichment results of the crucial 40 genes.

\begin{tabular}{lccll}
\hline Term & ID & Count & P-value & \multicolumn{1}{c}{ Genes } \\
\hline Protein processing in ER & hsa04141 & 5 & 0.0089 & FBXO2, DNAJC10, SSR3, CUL1, UBE2D3 \\
AMPK signaling pathway & hsa04152 & 4 & 0.0144 & PRKAB2, PFKP, PRKAA1, CREB1 \\
Dorso-ventral axis formation & hsa04320 & 2 & 0.0188 & MAPK1, NOTCH1 \\
Ubiquitin mediated proteolysis & hsa04120 & 4 & 0.0199 & FBXO2, CUL1, CUL7, UBE2D3 \\
Prion diseases & hsa05020 & 2 & 0.0313 & MAPK1, NOTCH1
\end{tabular}

ER, endoplasmic reticulum; AMPK, AMP-activated protein kinase; FBXO2, F-box protein 2; DNAJC10, DnaJ heat shock protein family (Hsp40) member C10; SSR3, signal sequence receptor 3; CUL1, cullin 1; UBE2D3, ubiquitin conjugating enzyme E2 D3; PRKAB2, protein kinase AMP-activated non-catalytic subunit $\beta$ 2; PFKP, phosphofructokinase, platelet; PRKAA1, protein kinase AMP-activated catalytic subunit $\alpha$ 1; CREB1, cAMP responsive element binding protein 1; MAPK1, mitogen-activated protein kinase 1; NOTCH1, notch 1; CUL7, cullin 7 :

suggesting that the alteration of this gene may affect the AMPK signaling pathway, whereby it may contribute to the metastasis of CRC. Based on this finding, it may be inferred that the CREB1-mediated AMPK signaling pathway has the potential to serve as a therapeutic marker for the diagnosis of CRC metastasis.

As a major component of the ubiquitin proteasome system, E3 ubiquitin ligases serve an important function in orchestrating the substrate ubiquitination in the cullin, Skp and F-box-containing complex (32). Disruption of their roles is the primary cause of the occurrence of various types of cancer (33). The CUL7 protein is a complex of the E3 ubiquitin-protein ligase that also comprises the S-phase kinase-associated protein 1, F-box/WD repeat-containing protein 8 (FBXW8) and E3 ubiquitin-protein ligase RBX1 proteins. Reportedly, the CUL7/FBXW8 complex inhibits cell growth in gastric cancer by inducing the expression of insulin receptor substrate 1 (34). The overexpression of CUL7 has been detected in hepatocellular carcinoma (HCC) tissues, particularly in metastatic $\mathrm{HCC}$, and in vitro experiments have demonstrated that the knockdown of this gene pronouncedly decreases the metastatic capacity of HCC (32). In addition, the expression of $C U L 7$ has been observed to be increased in non-small cell lung cancer cells, with its high expression potentially promoting the invasion and metastasis of these cells (35). Cyclin D1 is a vital protein for cell proliferation in various types of cancer. Its activation is controlled via the degradation caused by ubiquitin-mediated proteolysis (36). In the present study, CUL7 and its family member CUL1 were enriched in the ubiquitin-mediated proteolysis pathway, suggesting that their activation through this pathway may also regulate cell proliferation in CRC. However, there is insufficient evidence supporting the involvement of CUL7 in metastatic CRC. The results of the present study indicate that CUL7 is a signature gene that is able to distinguish between metastatic and non-metastatic CRC. In combination with the previously reported findings that $C U L 7$ is involved in the metastasis of other cancers, it is speculated that this gene may also be implicated in metastatic CRC, and is activated via the ubiquitin-mediated proteolysis pathway.

SSR is a glycosylated membrane receptor responsible for protein entry into the ER (37). As one of the four SSR family members, SSR3 is a non-glycosylated subunit that mediates the translocation of nascent polypeptide through the ER membrane (38). Reportedly, in pancreatic cancer (PAC) and prostate cancer, the expression of SSR3 is elevated, and the inhibition of this gene may weaken the potential tumor growth of PAC $(39,40)$. Furthermore, in a study using an RNA sequencing method, it was predicted that $S S R 3$ is a target of a long noncoding RNA, RP5-890E16.4, that may have crucial roles in esophageal squamous cell carcinoma (41). In murine breast tumor, SSR3 has been identified as a DEG associated with metastasis, using an exon-based clustering method (42). However, to the best of our knowledge, no further information is available concerning the role of this gene in CRC progression, particularly in metastasis. In the present study, SSR3 was demonstrated to be a critical gene signature of metastatic CRC that was enriched in the protein processing in ER pathway. Together, the aforementioned information suggests that SSR3 may serve an important function in the metastasis of CRC via involvement in the protein processing in ER pathway, and could be used as a novel therapeutic target for the treatment of metastatic CRC.

Despite these comprehensive analyses and the precision of the SVM classifier, the present study has the limitation that all the predicted results lack experimental validation. Nevertheless, the findings are valuable as they provide novel insights into the regulatory mechanisms of the metastasis of CRC and identify novel biomarkers for the prognosis of this disease.

In conclusion, the SVM-classified gene signatures in the present study precisely distinguished metastatic CRC samples from non-metastatic ones, using genes including $C R E B 1$, $C U L 7$ and SSR3. The genes could be used as biomarkers for the prognosis of metastatic CRC. However, substantial additional experiments are required to validate the predicted expression levels and functions.

\section{References}

1. Haggar FA and Boushey RP: Colorectal cancer epidemiology: Incidence, mortality, survival, and risk factors. Clin Colon Rectal Surg 22: 191-197, 2009.

2. Siegel R, Desantis C and Jemal A: Colorectal cancer statistics, 2014. CA Cancer J Clin 64: 104-117, 2014. 
3. Zhang B, Jia WH, Matsuda K, Kweon SS, Matsuo K, Xiang YB, Shin A, Jee SH, Kim DH, Cai Q, et al; Genetics and Epidemiology of Colorectal Cancer Consortium (GECCO); Colorectal Transdisciplinary (CORECT) Study; Colon Cancer Family Registry (CCFR): Large-scale genetic study in East Asians identifies six new loci associated with colorectal cancer risk. Nat Genet 46: 533-542, 2014.

4. Zhang W, Glöckner SC, Guo M, Machida EO, Wang DH, Easwaran H, Van Neste L, Herman JG, Schuebel KE, Watkins DN, et al: Epigenetic inactivation of the canonical Wnt antagonist SRY-box containing gene 17 in colorectal cancer. Cancer Res 68: 2764-2772, 2008.

5. Kunnumakkara AB, Diagaradjane P, Guha S, Deorukhkar A, Shentu S, Aggarwal BB and Krishnan S: Curcumin sensitizes human colorectal cancer xenografts in nude mice to gammaradiation by targeting nuclear factor-kappaB-regulated gene products. Clin Cancer Res 14: 2128-2136, 2008.

6. Chaffer CL and Weinberg RA: A perspective on cancer cell metastasis. Science 331: 1559-1564, 2011.

7. Di Nicolantonio F, Martini M, Molinari F, Sartore-Bianchi A, Arena S, Saletti P, De Dosso S, Mazzucchelli L, Frattini M, Siena $\mathrm{S}$, et al: Wild-type BRAF is required for response to panitumumab or cetuximab in metastatic colorectal cancer. J Clin Oncol 26: 5705-5712, 2008.

8. Rychahou PG, Kang J, Gulhati P, Doan HQ, Chen LA, Xiao SY, Chung DH and Evers BM: Akt2 overexpression plays a critical role in the establishment of colorectal cancer metastasis. Proc Natl Acad Sci USA 105: 20315-20320, 2008

9. Jorissen RN, Gibbs P, Christie M, Prakash S, Lipton L, Desai J, Kerr D, Aaltonen LA, Arango D, Kruhøffer M, et al: Metastasis-associated gene expression changes predict poor outcomes in patients with dukes stage B and C colorectal cancer. Clin Cancer Res 15: 7642-7651, 2009.

10. Smith JJ, Deane NG, Wu F, Merchant NB, Zhang B, Jiang A, Lu P, Johnson JC, Schmidt C, Bailey CE, et al: Experimentally derived metastasis gene expression profile predicts recurrence and death in patients with colon cancer. Gastroenterology 138 958-968, 2010

11. Ben-Hur A and Weston J: A user's guide to support vector machines. Humana Press, 2010.

12. Mourao-Miranda J, Reinders AA, Rocha-Rego V, Lappin J, Rondina J, Morgan C, Morgan KD, Fearon P, Jones PB, Doody GA, et al: Individualized prediction of illness course at the first psychotic episode: A support vector machine MRI study. Psychol Med 42: 1037-1047, 2012.

13. Henneges C, Bullinger D, Fux R, Friese N, Seeger H, Neubauer H, Laufer S, Gleiter CH, Schwab M, Zell A, et al: Prediction of breast cancer by profiling of urinary RNA metabolites using support vector machine-based feature selection. BMC Cancer 9 $104,2009$.

14. Gabere MN, Hussein MA and Aziz MA: Filtered selection coupled with support vector machines generate a functionally relevant prediction model for colorectal cancer. Onco Targets Ther 9: 3313-3325, 2016.

15. Gross AM, Kreisberg JF and Ideker T: Analysis of matched tumor and normal profiles reveals common transcriptional and epigenetic signals shared across cancer types. PLoS One 10: e0142618, 2015.

16. Del Rio M, Mollevi C, Vezzio-Vie N, Bibeau F, Ychou M and Martineau P: Specific extracellular matrix remodeling signature of colon hepatic metastases. PLoS One 8: e74599-e74599, 2013.

17. Lin AY, Chua MS, Choi YL, Yeh W, Kim YH, Azzi R, Adams GA, Sainani K, van de Rijn M, So SK, et al: Comparative profiling of primary colorectal carcinomas and liver metastases identifies LEF1 as a prognostic biomarker. PLoS One 6: e16636, 2011.

18. Stange DE, Engel F, Longerich T, Koo BK, Koch M, Delhomme N, Aigner M, Toedt G, Schirmacher P, Lichter P, et al: Expression of an ASCL2 related stem cell signature and IGF2 in colorectal cancer liver metastases with 11p15.5 gain. Gut 59: 1236-1244, 2010.

19. Ki DH, Jeung HC, Park CH, Kang SH, Lee GY, Lee WS Kim NK, Chung HC and Rha SY: Whole genome analysis for liver metastasis gene signatures in colorectal cancer. Int J Cancer 121: 2005-2012, 2007.

20. Cho WK, Geimer S and Meurer J: Cluster analysis and comparison of various chloroplast transcriptomes and genes in Arabidopsis thaliana. DNA Res 16: 31-44, 2009.

21. Bolstad BM, Irizarry RA, Astrand M and Speed TP: A comparison of normalization methods for high density oligonucleotide array data based on variance and bias. Bioinformatics 19: 185-193, 2003.
22. Smyth GK: limma: Linear models for microarray data. In: Bioinformatics and Computational Biology Solutions Using R and Bioconductor. Gentleman R, Carey VJ, Huber W, Irizarry RA and Dudoit S (eds). Statistics for Biology and Health. Springer, New York, NY, 2005.

23. Kang DD, Sibille E, Kaminski N and Tseng GC: MetaQC: Objective quality control and inclusion/exclusion criteria for genomic meta-analysis. Nucleic Acids Res 40: e15, 2012.

24. Keshava Prasad TS, Goel R, Kandasamy K, Keerthikumar S, Kumar S, Mathivanan S, Telikicherla D, Raju R, Shafreen B, Venugopal A, et al: Human Protein Reference Database - 2009 update. Nucleic Acids Res 37: D767-D772, 2009.

25. Stark C, Breitkreutz BJ, Chatr-Aryamontri A, Boucher L, Oughtred R, Livstone MS, Nixon J, Van Auken K, Wang X, Shi X, et al: The BioGRID Interaction Database: 2011 update. Nucleic Acids Res 39: D698-D704, 2011.

26. Kintali S: Betweenness Centrality : Algorithms and Lower Bounds. Comput Sci, 0809.1906v2, 2008.

27. Guyon I, Weston J, Barnhill S and Vapnik V: Gene Selection for Cancer Classification using Support Vector Machines. Mach Learn 46: 389-422, 2002.

28. Li P, Xue WJ, Feng Y and Mao QS: MicroRNA-205 functions as a tumor suppressor in colorectal cancer by targeting cAMP responsive element binding protein 1 (CREB1). Am J Transl Res 7: 2053-2059, 2015.

29. Wang YW, Chen X, Ma R and Gao P: Understanding the CREB1-miRNA feedback loop in human malignancies. Tumour Biol 37: 8487-8502, 2016

30. Tong W, Sun D, Wang Q and Suo J: Sorcin enhances metastasis and promotes epithelial-to-mesenchymal transition of colorectal cancer. Cell Biochem Biophys 72: 453-459, 2015.

31. Hwang JT, Kwak DW, Lin SK, Kim HM, Kim YM and Park OJ: Resveratrol induces apoptosis in chemoresistant cancer cells via modulation of AMPK signaling pathway. Ann NY Acad Sci 1095: 441-448, 2007.

32. Zhang D, Yang G, Li X, Xu C and Ge H: Inhibition of liver carcinoma cell invasion and metastasis by knockdown of cullin7 in vitro and in vivo. Oncol Res 23: 171-181, 2016.

33. Satija YK, Bhardwaj A and Das S: A portrayal of E3 ubiquitin ligases and deubiquitylases in cancer. Int J Cancer 133: 2759-2768, 2013

34. Chen P and Yao GD: The role of cullin proteins in gastric cancer. Tumour Biol 37: 29-37, 2016.

35. Song Q, Wang L, Lu Y, Zhang J and Fu J: Abstract 2008: CUL7 promotes non-small cell lung cancer cells migration and invasion. Cancer Res 74 (Suppl 19): 2008, 2014.

36. Achiwa Y, Hasegawa K and Udagawa Y: Effect of ursolic acid on MAPK in cyclin D1 signaling and RING-type E3 ligase (SCF E3s) in two endometrial cancer cell lines. Nutr Cancer 65: 1026-1033, 2013

37. Wang L and Dobberstein B: Oligomeric complexes involved in translocation of proteins across the membrane of the endoplasmic reticulum. FEBS Lett 457: 316-322, 1999.

38. Chen HZ, Wen Q, Wang WJ, He JP and Wu Q: The orphan nuclear receptor TR3/Nur77 regulates ER stress and induces apoptosis via interaction with TRAP $\gamma$. Int J Biochem Cell Biol 45: 1600-1609, 2013

39. Dehm SM and Tindall DJ: Molecular regulation of androgen action in prostate cancer. J Cell Biochem 99: 333-344, 2006.

40. Chen Y, Jesnowski R and Löhr JM: Cloning and characterization of genes differentially expressed in human pancreatic carcinoma. Z Gastroenterol: 43, 2005.

41. Li Y, Shi X, Yang W, Lu Z, Wang P, Chen Z and He J: Transcriptome profiling of lncRNA and co-expression networks in esophageal squamous cell carcinoma by RNA sequencing. Tumour Biol 37: 13091-13100, 2016.

42. Dutertre M, Lacroix-Triki M, Driouch K, de la Grange P, Gratadou L, Beck S, Millevoi S, Tazi J,Lidereau R, Vagner S, et al: Exon-based clustering of murine breast tumor transcriptomes reveals alternative exons whose expression is associated with metastasis. Cancer Res 70: 896-905, 2010.

This work is licensed under a Creative Commons Attribution-NonCommercial-NoDerivatives 4.0 International (CC BY-NC-ND 4.0) License. 